\title{
Implementation of a systematic tobacco treatment protocol in a surgical outpatient setting: a feasibility study
}

\author{
Joseph Sadek, MD \\ Husein Moloo, MD \\ Priscilla Belanger, MSc \\ Kara Nadeau, MD \\ Debbie Aitken, BScN \\ Kris Foss, BScN \\ Terry Zwiep, MD \\ Dan Mclsaac, MD \\ Lara Williams, MD \\ Isabel Raiche, MD \\ Reily Musselman, MD \\ Kerri-Anne Mullen, PhD
}

Previously presented at the American College of Surgeons Quality and Safety Conference, the BMJ International Forum on Quality and Safety in Healthcare and the Canadian Surgical Forum.

Accepted March 6, 2020

\author{
Correspondence to: \\ H. Moloo \\ Division of General Surgery \\ Faculty of Medicine, University of Ottawa \\ 305-737 Parkdale Ave. \\ Ottawa ON K1J 4E9 \\ hmoloo@toh.ca
}

DOI: $10.1503 /$ cjs.009919

\begin{abstract}
Background: Smoking cessation programs started as late as 4 weeks before surgery reduce perioperative morbidity and death, yet outpatient clinic interventions are rarely provided. Our aim was to evaluate the feasibility of implementing a tobacco treatment protocol designed for an outpatient surgical setting.
\end{abstract}

Methods: We completed a pre-post feasibility study of the implementation of a systematic, evidence-based tobacco treatment protocol in an outpatient colorectal surgery clinic. Outcomes included smoking prevalence, pre- and postimplementation smoker identification and intervention rates, recruitment, retention, smoking cessation and provider satisfaction.

Results: Preimplementation, $15.5 \%$ of 116 surveyed patients were smokers. Fewer than $10 \%$ of surveyed patients reported being asked about smoking, and none were offered any cessation intervention. Over a 16-month postimplementation period, 1198 patients were seen on 2103 visits. Of these, 950 (79.3\%) patients were asked smoking status on first visit and $1030(86.0 \%)$ were asked on at least 1 visit. Of 169 identified smokers, $99(58.6 \%)$ were referred to follow-up support using an opt-out approach. At 1-, 3- and 6-month follow-up, intention-to-quit rates among 78 enrolled patients were $24.4 \%, 22.9 \%$ and $19.2 \%$, respectively. Postimplementation staff surveys reported that the protocol was easy to use, that staff would use it again and that it had positive patient responses.

Conclusion: Implementation of our smoking cessation protocol in an outpatient surgical clinic was found to be feasible and used minimal clinic resources. This protocol could lead to increases in identification and documentation of smoking status, delivery of smoking cessation interventions and rates of smoking reduction and cessation.

Contexte : Les programmes d'abandon du tabagisme entamés jusqu'à 4 semaines avant une opération réduisent la morbidité et la mortalité périopératoires, mais les cliniques externes n'en proposent que rarement. L'étude visait à évaluer s'il est faisable d'appliquer un protocole de traitement du tabagisme pensé pour les milieux de soins chirurgicaux extrahospitaliers.

Méthodes : Nous avons réalisé une étude de faisabilité pré- et postexpérimentale sur l'application d'un protocole de traitement systématique fondé sur des données probantes à une clinique externe de chirurgie colorectale. Les résultats à l'étude étaient les suivants : prévalence du tabagisme, identification des fumeurs et taux d'intervention avant et après la mise en place du protocole, recrutement, rétention, abandon du tabagisme et satisfaction des fournisseurs.

Résultats : Au départ, 15,5\% des 116 patients sondés fumaient. Moins de $10 \%$ des répondants avaient été questionnés sur leur statut tabagique, et aucun ne s'était vu proposer un programme d'abandon. Au cours des 16 mois suivant la mise en place du protocole, 1198 patients ont été rencontrés dans le cadre de 2103 consultations. Parmi eux, $950(79,3 \%)$ ont été interrogés sur leur statut tabagique à la première rencontre, et 1030 (86\%) l'ont été au moins 1 fois. Des 169 fumeurs identifiés, 99 (58,6\%) ont été orientés vers un programme de soutien selon une approche de consentement présumé. Après 1 mois, 24,4\% des 78 patients participants étaient déterminés à arrêter de fumer; 22,9\% l'étaient toujours après 3 mois, et 19,2\% après 6 mois. Les sondages menés a posteriori auprès du personnel indiquent que le protocole est facile à utiliser, que les employés s'en serviraient de nouveau, et que les patients l'ont accueilli favorablement.

Conclusion : Il a été possible de mettre en place notre protocole d'abandon du tabagisme à une clinique externe de chirurgie, et ce en employant un minimum de ressources cliniques. Le protocole pourrait permettre de connaître et de consigner davantage de statuts tabagiques, d'orienter un plus grand nombre de fumeurs vers les programmes d'abandon et d'accroître les taux de réduction et d'abandon du tabagisme. 
D espite decreased smoking rates in recent decades, smoking remains the leading cause of preventable disease and death in Canada. ${ }^{1}$ In 2017, 15\% of Canadians older than age 15, roughly 4.6 million people, were smokers. ${ }^{2}$ Among some inpatient hospital populations, the smoking prevalence is even higher (> 20\%). ${ }^{3}$ Smoking is a known risk factor for perioperative complications. In a retrospective cohort study of more than 600000 surgical patients, preoperative smoking was associated with a $40 \%$ increased odds of 30-day mortality (odds ratio [OR] 1.38, $95 \%$ confidence interval $[\mathrm{CI}] 1.11-1.72)$ and a $70 \%$ increased odds of major morbidity (OR 1.72, 95\% CI 1.671.78), including pneumonia, unplanned intubation, cardiac arrest, myocardial infarction, stroke, organ space infections and sepsis. ${ }^{4}$ Not only is this troublesome for patients, but it also leads to increased costs to the health care system. ${ }^{5}$ Smoking is a known risk factor in most surgeries, with higher rates of incisional hernia after laparotomy (OR 3.93, 95\% CI 1.82-8.49), ${ }^{6}$ spinal fusion nonunion (OR 2.01, $p<0.016),{ }^{7}$ disease recurrence (hazard ratio [HR] 1.25, $p=0.024)$ and metastases (HR 2.64, $p=0.026)$ after radical prostatectomy, ${ }^{8}$ anastomotic leakage after anterior resection (OR 1.88, 95\% CI 1.02-3.46), ${ }^{9}$ and fracture after shoulder arthroplasty (HR 3.63, $p=0.025)^{10}$ Even among nonoperative patients, smoking cessation has countless benefits including improved cardiorespiratory function, ${ }^{11,12}$ reduced rates of numerous cancers, ${ }^{13,14}$ increases in life expectancy ${ }^{14,15}$ and fewer admissions to hospital, with shorter lengths of stay and lower health care costs. ${ }^{16}$

Two landmark randomized controlled trials (RCTs) showed improved postsurgical outcomes with preoperative smoking cessation programs, involving intensive behavioural interventions and nicotine replacement therapy. One program, initiated 6-8 weeks before hip or knee surgery, led to significant reductions in overall $(18 \%$ v. $52 \%$, $p<0.001)$ and wound-related (5\% v. $31 \%, p=0.001)$ complications. ${ }^{17}$ Another program, with a target smoking cessation date of 4 weeks before general or orthopedic surgery, showed significant reduction in any postoperative complication $(21 \%$ v. $41 \%$, relative risk [RR] 0.51, $p=0.03) .{ }^{18}$ More recently, an observational, nested, matched case-control study showed a benefit to quitting as late as the day of surgery, showing a significant increase in surgical site infections in active smokers (OR 1.96, 95\% CI $1.23-3.13, p<0.001){ }^{19}$

Smoking is believed to cause numerous physiologic changes including temporary reduction in tissue perfusion and oxygenation, impairment of inflammatory cell function and oxidative bactericidal mechanisms, and attenuation of reparative cell functions including collagen synthesis and deposition. These all appear to contribute to surgical complications and are thought to improve to varying degrees based on duration since cessation. ${ }^{20}$ Despite the overwhelming evidence of the negative effects of tobacco smoking and the fact that roughly 1 in 4 surgical patients are smokers, ${ }^{4}$ more than half of elective outpatient surgical patients report not being informed about the adverse effects of smoking before surgery. ${ }^{21}$

The overall aim of our pilot study was to evaluate the feasibility, potential effectiveness, and implementation of a practical, evidence-based, opt-out tobacco treatment protocol, as implemented at an outpatient surgery clinic in Ottawa, Ontario. The primary objectives were to evaluate feasibility outcomes (i.e., recruitment and retention) by determining the prevalence of current smoking among surgery clinic patients, the number of smokers referred for tobacco treatment support, and the number of smokers opting in to that support. Secondary objectives were to determine the potential effectiveness of the intervention by measuring individual smoking rates. Implementation outcomes included the number of patients asked about smoking status and the number of smokers who were treated and referred to follow-up.

\section{Methods}

\section{Study design, setting and participants}

We completed a pre-post feasibility study of the implementation of a systematic, evidence-based tobacco treatment protocol in an outpatient surgical clinic. Components of the RE-AIM (reach, effectiveness, adoption, implementation, maintenance) framework were used to measure feasibility, effectiveness and implementation outcomes. ${ }^{22}$

The study took place at The Ottawa Hospital in an outpatient colorectal surgery clinic between November 2016 and February 2018. Annually, the clinic has 1600 adult patient visits for issues primarily related to colorectal cancer, inflammatory bowel disease and perianal disease. Patients can be in any stage of their care (preoperative, postoperative or being medically managed), and often a patient has multiple visits throughout the year. The clinic is staffed with 2 full-time nurse specialists, 4 surgeons, 1 or 2 rotating residents and an administrative assistant. Following the clinic intervention, phone follow-up was done through the Ottawa Model for Smoking Cessation (OMSC).

\section{Protocol development and implementation}

A multidisciplinary team of stakeholders including surgeons, residents, clinic nurses, a medical student, an anesthetist and several implementation specialists from the OMSC met between October 2015 and October 2016 to develop a tobacco treatment protocol suited for fast-paced outpatient surgery clinics. A novel protocol, preprinted prescription and assessment form (see Appendix 1, available at canjsurg.ca/009919-a1) were designed with speed and ease of use in mind. 
The protocol began with systematic identification of smoking status of all patients. For all clinic visits, the clinic nurse took a brief appointment-related initial patient history. Based on the patient's response to the question: "Have you used any form of tobacco in the past 6 months?", the nurse checked off the appropriate response in the "Smoker: Yes/No" box, stamped in advance on the standard clinic encounter sheets. Identified smokers were given an assessment form regarding their smoking, including components of the Fagerstrom questionnaire (e.g., number of cigarettes per day, duration of smoking, how soon after waking first cigarette is smoked), and intentions and readiness to quit. Once the patient was identified as a smoker, one of the involved clinicians (nurse, resident, or surgeon) would offer the smoking cessation intervention, typically at the end of the clinic visit. The clinician briefly reviewed the information filled in by the patient and completed the rest of the form, which prompted them to provide the following to the patient: encouragement regarding cessation and its benefits, a prescription for nicotine replacement therapy, a smoking cessation education booklet and a referral to smoking cessation follow-up support. This intervention was designed to be practical in a rapid outpatient surgical clinic, as we know that interventions that are time consuming or cumbersome are rarely sustainable and often ignored altogether. For each additional minute required for the intervention, we anticipated that implementation rates would drop. Given the evidence that brief intervention is effective ${ }^{23}$ and that the single most effective preoperative behavioural intervention to induce cessation is informing patients about the consequences of smoking and cessation, ${ }^{24}$ we elected to focus clinician time in this area.

Smoking cessation follow-up support was offered using an opt-out approach, whereby patients were informed that they would be referred for follow-up telephone counselling from a tobacco treatment nurse specialist (TTNS) at the OMSC program, unless they actively refused. The patient smoking assessment form was faxed to the OMSC program, and a TTNS called patients within 7 days of their referral to provide more detailed counselling and explain the follow-up program. Enrolled patients received a further 5 automated calls over 6 months. Calls included prerecorded questions about current smoking status and confidence with regard to quitting; patients responded using their telephone keypad. Certain responses by the patient (e.g., relapse, low confidence) prompted a followup call from a TTNS for further counselling. Each automated call ended with a motivational message and a reminder of when to expect their next call.

\section{Outcomes}

Before implementing the new protocol, we measured the preimplementation prevalence of smoking among clinic patients and what, if any, tobacco treatment interventions were being offered by clinic staff. A cohort of patients was given a 4-question survey by a medical student on random days between July and December 2016 immediately following their clinic visit with a nurse or staff surgeon who were not aware of the survey. The patients could be in any phase of care: new consult, follow-up, preoperation or postoperation. On each assessment day, a consecutive series of patients were asked about current smoking status and whether their smoking status had been queried during their appointment. Patients who were current smokers were then asked whether smoking cessation had been discussed during their visit and whether any cessation intervention had been offered. Clinical practice guidelines suggest that effective treatment of smoking requires consistent identification of smokers at every health care encounter. ${ }^{23}$ For this reason, survey responses were based on this specific clinic visit and did not include whether the patient had been asked smoking status on a previous visit. The aim of this questionnaire was to identify how consistently smoking was being identified and treated on a random clinic visit, not whether staff had documented smoking status at some point in the past.

Postimplementation, the proportion of patients who were queried about their tobacco use was determined via chart audit by reviewing the "Smoker: Yes/No" stamp on the encounter sheet. As this method was developed for the new protocol, a similar chart audit was not possible preimplementation.

Feasibility outcomes included smoking cessation intervention, recruitment and retention rates. The rate of smoking cessation intervention was defined as the proportion of patient-smokers that were offered tobacco treatment follow-up support, as identified on chart audit. This rate included both patients who subsequently refused the offered intervention as well as patients who were then referred to the OMSC. Recruitment was defined as the proportion of patient-smokers identified on chart audit that agreed to tobacco treatment follow-up support and was measured as the number of patients referred and registered in the OMSC online system. Retention was measured as the proportion of smokers referred to the OMSC follow-up system who then opted in to long-term support at the time of the initial phone call with the TTNS.

As an estimate of the effectiveness of the intervention at changing smoking behaviours, self-reported pointprevalence quit rates were gathered at 1 month, 3 months and 6 months after referral to the OMSC, based on participant response to the question "Have you used any form of tobacco in the past 7 days?" The Russell standard was used, whereby all participants were used in the analysis, minus those who had moved to an untraceable address or whose telephone was no longer in service. ${ }^{25}$ If a patient was otherwise unreachable (e.g., did not answer phone/refused), they 
were considered smokers for the purposes of the analysis. To assess reductions in smoking, patients who were still smoking at follow-up assessments were asked how many cigarettes they currently smoked per day, which was then subtracted from the number smoked per day at baseline.

Finally, to determine the feasibility of uptake among the health providers in the clinic, clinic staff completed an anonymous 11-question online survey about the intervention (Appendix 1).

\section{Statistical analysis}

Data were summarized using descriptive statistics.

\section{Ethics}

The program was reviewed by The Ottawa Hospital Research Ethics Board. The evaluation activities published herein were deemed not to be research, but rather considered as quality improvement processes.

\section{Results}

\section{Pre- and postimplementation prevalence of smokers}

A total of 116 consecutive colorectal surgery clinic patients were surveyed preimplementation (July to December 2016), with no patients excluded. Of these, 18 patients $(15.5 \%)$ were current smokers and $10(8.6 \%)$ had been asked their smoking status. Among the smokers, none were asked about quitting, nor were they offered any type of tobacco treatment intervention.

Postimplementation, 1198 patients were seen for 2103 visits over a 16-month period, from November 1, 2016, to February 28, 2018 (Figure 1). In that time, 710 patients were seen only once, 276 were seen twice, 106 were seen 3 times and 106 were seen between 4 and 10 times. A total of $950(79.3 \%)$ patients were asked about their smoking status at their first visit and $1030(86.0 \%)$ were asked on at least 1 visit. Of these, $169(16.4 \%)$ patients were identified as smokers.

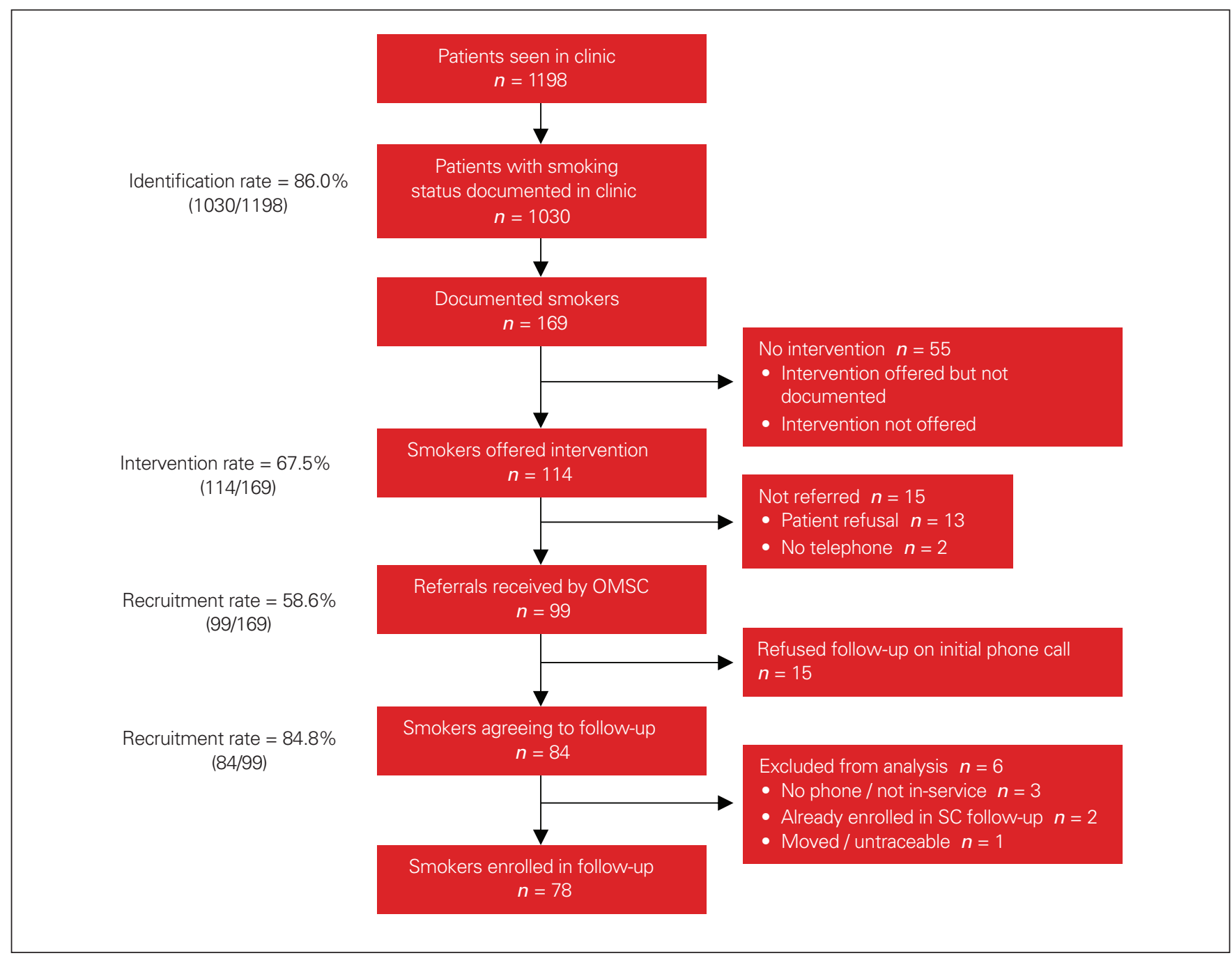

Fig. 1. Patient flowchart. OMSC = Ottawa Model for Smoking Cessation, $\mathrm{SC}=$ smoking cessation. 


\section{Feasibility outcomes}

Of the 169 unique identified smokers, 114 (67.5\%) were offered the in-clinic smoking cessation intervention. Of the 114,99 patients $(86.8 \%)$ were referred on to smoking cessation follow-up support using the opt-out approach, and 60 patients $(52.6 \%)$ were prescribed a smoking cessation medication $50.0 \%$ combination nicotine replacement therapy, $2.6 \%$ varenicline). The retention rate was $73.7 \%$, with 84 of the 114 patients referred to follow-up support agreeing to participate in long-term follow-up. Six of these patients did not ultimately enrol, thus 78 patients participated in long-term follow-up. Table 1 shows the baseline characteristics of the patients who enrolled in smoking cessation follow-up.

\section{Changes in smoking behaviour}

Figure 2 summarizes smoking behaviour outcomes among the 78 patients who participated in long-term follow-up. Intention-to-quit (nonsmoker) rates at 1, 3 and 6 months were $24.4 \%, 23.1 \%$ and $19.2 \%$, respectively. Nonresponders made up 7 (9.0\%), 11 (14.1\%)

Table 1. Baseline characteristics of enrolled patients $(n=78)$

\begin{tabular}{|lc|}
\hline Variable & No. (\%) of patients* \\
\hline Sex, male & $46(59.0)$ \\
\hline Age, yr, mean \pm SD & $50.2 \pm 12.2$ \\
\hline Years smoking, mean \pm SD & $28.5 \pm 14.0$ \\
\hline Cigarettes per day, mean \pm SD & $16.6 \pm 10.5$ \\
\hline $\begin{array}{l}\text { Minutes to first cigarette after waking, mean } \pm \\
\text { SD }\end{array}$ & $40.5 \pm 85.8$ \\
\hline Ready to quit in next 30 days & $41(52.3)$ \\
\hline $\begin{array}{l}\text { SD }=\text { standard deviation. } \\
\text { *Unless indicated otherwise. }\end{array}$ \\
\hline
\end{tabular}

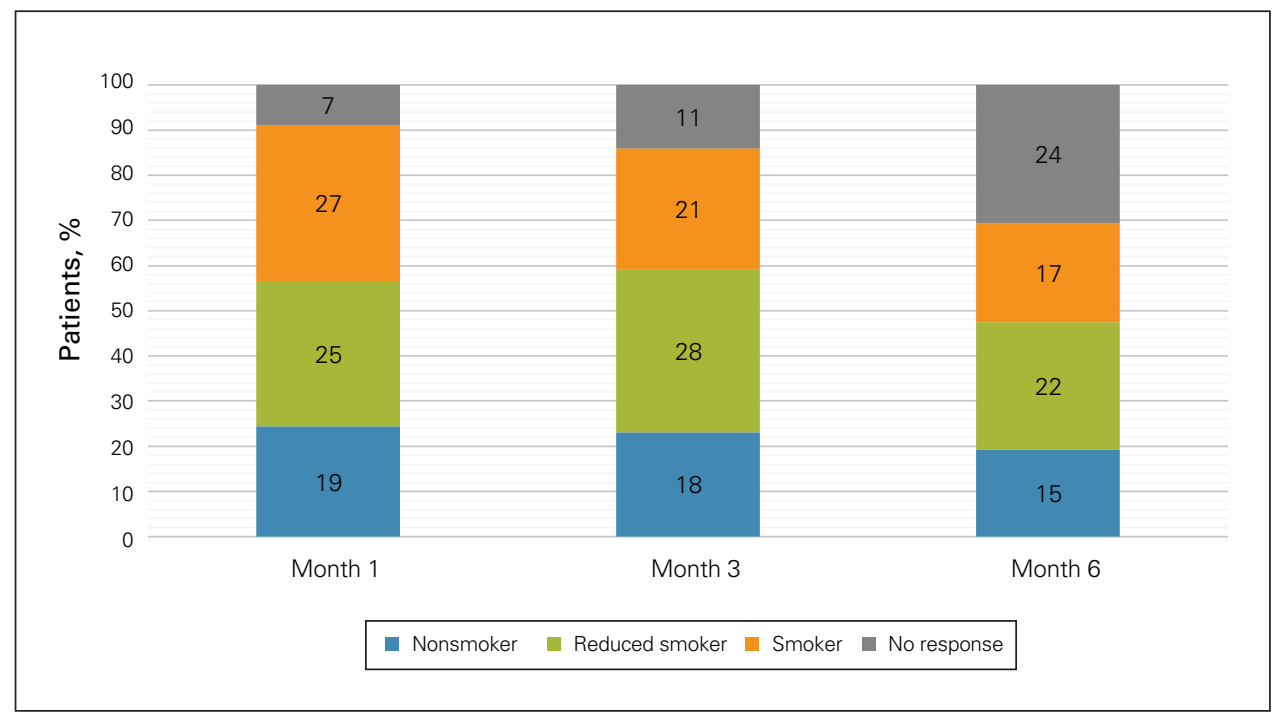

Fig. 2. Smoking behaviour outcomes among patients who participated in long-term follow-up with the Ottawa Model for Smoking Cessation program. and $24(30.8 \%)$ of calls; thus, quit rates among responders were of $26.8 \%$ (19 of 71 responders), $26.9 \%$ (18 of 67 responders), and $27.8 \%$ (15 of 54 responders), respectively. Twenty-five (32.1\%), 28 (35.9\%) and 22 $(28.2 \%)$ patients reported reducing the amount they smoked at 1, 3 and 6 months, respectively. Their mean (standard deviation) reductions at each time point in the number of cigarettes smoked per day were $9.2(7.3), 9.8$ (8.2) and 7.5 (7.1) respectively.

\section{Health provider feedback}

Ten surgeons, residents and nurses who used the protocol completed the anonymous 11-question survey. Preimplementation, clinicians very rarely offered smoking cessation interventions, citing barriers such as lack of time, lack of knowledge and inability to follow up. After using the protocol, these barriers were greatly reduced. Every clinician felt the intervention was easy to use, would use it again and would recommend it to other health care workers in their clinic. All felt that patients responded positively. On average, the intervention took 4-6 minutes. Postimplementation, clinicians were more likely to address smoking both in the clinic and in other settings.

\section{Discussion}

This evaluation tested the feasibility of a smoking cessation protocol designed for fast-paced outpatient surgical clinics, as implemented in a colorectal surgery clinic. Following implementation of the protocol, the rate of smoking status identification increased from $8.6 \%$ to $86.0 \%$. The rate of providing an initial tobacco treatment intervention to smokers in the clinic increased from $0 \%$ to $67.5 \%$. Preimplementation identification and intervention rates were dramatically low, highlighting the importance of this work. It should be noted these numbers reflect a snapshot of that specific visit, and it is possible that smoking status had previously been documented. However, smoking status can change and should be documented at every health care encounter. ${ }^{23}$ Postimplementation, the reason identification and intervention rates were not $100 \%$ is likely a combination of a failure to document all interventions which were offered but declined, in which case the true number is actually higher, and true misses from human error in a busy clinic. 
More than $50 \%$ of smokers who received the in-clinic tobacco treatment intervention were prescribed a smoking cessation medication, and nearly $60 \%$ of identified smokers were referred to smoking cessation follow-up support using an opt-out approach. Half of all smokers identified in clinic remained enrolled at 6-month follow-up.

Cessation rates at 1- (24.4\%), 3- (23.1\%) and 6-month (19.2\%) follow-up are promising. Although we did not have spontaneous quit rates for the preimplementation group for direct comparison, 2 perioperative smoking cessation RCTs showed control group cessation rates of $1.6 \%{ }^{26}$ and $3.6 \%,{ }^{27}$ and the unassisted cessation rate in the general public is less than $2 \% .{ }^{28}$ In our study, more than $40 \%$ of nonquitters smoked an average of nearly 10 fewer cigarettes per day compared to baseline. Recent evidence suggests that reduction strategies do increase the odds of eventual cessation, even for smokers with no initial intention of full cessation. ${ }^{29}$

Two previous RCTs showing improved perioperative outcomes with smoking cessation programs both used intensive interventions, including weekly meetings with a trained nurse. ${ }^{17,18}$ With the additional costs and time requirements, this is not always feasible in the realworld setting. Our program was designed to use existing clinic resources and add no more than 5 minutes to the clinic visit.

Patients had a faxed referral sent to an existing clinical follow-up support program within the same health care system, requiring no additional funding. Although this specific OMSC program is not available nationally, every Canadian province has free programs available for phone follow-up of smokers. These resources are easily found on the Government of Canada website. ${ }^{30}$

For smokers who were seen in clinic, using an opt-out approach of providing cessation follow-up, rates of participation in long-term follow-up were drastically higher than that observed with "opt-in" approaches among hospitalized patients ( $84.8 \%$ v. $23 \%) .{ }^{31}$ Opt-out approaches to smoking cessation interventions are recommended in acute care settings. ${ }^{32}$ Given the impact of smoking cessation on surgical outcomes, opt-out approaches to offering tobacco treatment should become the default, as with the treatment of other clinical conditions known to affect outcomes. For example, when a preoperative patient reports having chest pain, we do not ask them if they would be interested in referral to a cardiologist. We simply tell them they are being referred. We should not see smoking as a habit to be optionally addressed, but instead a serious medical issue to be systematically treated on every encounter.

Preoperative timing of cessation is a critical factor. Cessation, even on the day of surgery, has been shown to reduce wound complications. ${ }^{19}$ The earlier patients quit, the greater the postoperative benefit, with some studies suggesting that quitting more than 8 weeks preoperation leads to complication rates similar to that of nonsmokers. ${ }^{33,34}$ A Cochrane review of several RCTs with brief interventions targeting quit dates within 1 week of surgery showed significant reductions in smoking but not perioperative complications; however, with such short preoperative durations of cessation, along with small sample sizes, these results are not surprising. ${ }^{35}$ In another RCT, patients were seen in the anesthesia preadmission clinic at least 3 weeks preoperatively, and those who were randomized to intervention received brief counselling, a brochure, 6 weeks of free nicotine replacement therapy and referral to the Canadian Cancer Centre's Smokers Helpline. Smoking cessation rate in the intervention group was $14.3 \%$, compared to $3.6 \%$ in the. control group (RR 4.0, 95\% CI $1.2-$ $13.7, p=0.03)$, and at 30 days postoperation, these rates were $28.6 \%$ v. $11 \%$ (RR 2.6, 95\% CI, $1.2-5.5, p=0.008) .{ }^{27}$ However, more than two-thirds of patients were ineligible to participate in the study because preadmission clinic visits often take place too near the time of surgery.

The initial surgical clinic visit is the ideal time to intervene. Patients are typically seen in surgical clinics and booked for an operation several weeks before they arrive to the preadmission clinic, and often several months before their actual surgery date. As previously described, evidence suggests improved postoperative outcomes occur with earlier cessation. With sustained cessation rates at the 3 -month follow-up, our smoking cessation program would fit this timeline well.

\section{Limitations}

Our pilot study has several limitations. It is a pre-post cohort study lacking randomization or a control group. Cessation data are based on patient self-reported cessation, with no biochemical validation (i.e., plasma cotinine or expired carbon monoxide) because of prohibitive costs. We were, however, conservative in our assessment, and assumed that those unreachable for follow-up had resumed smoking. We did not record or stratify the intervention results by clinician (nurse, resident or surgeon) preventing us from analyzing the performance of individual clinicians within our clinic. Duration of intervention was measured only by clinician survey, which is subject to recall bias. For the 55 identified smokers who did not receive referral to the OMSC, we could not document whether referral was offered and refused versus not offered at all. Lastly, a large proportion of patients were lost to follow-up at 6 months.

\section{Conclusion}

We have shown that our smoking cessation protocol is feasible to implement in fast-paced surgical clinics using minimal resources, and can potentially lead to increases in smoking identification, documentation, and intervention. Subsequently this can translate to high rates of smoking 
reduction and cessation at 1-, 3- and 6-month follow-up. Our patient population had been smoking on average almost 1 pack per day for 28 years, and after this 5 -minute intervention, about a quarter quit smoking and another third reduced smoking by over $50 \%$. These are striking results given the highly addictive nature of smoking. ${ }^{36}$

Future studies should examine how timing of the intervention in relation to surgery can impact outcomes, which intervention components are most effective at helping people quit smoking and whether reduce-to-quit strategies lead to smoking cessation at the time of surgery.

Affiliations: From the Department of General Surgery, University of Ottawa Faculty of Medicine, Ottawa, Ont. (Sadek, Moloo, Zwiep, Williams, Raiche, Musselman); the Ottawa Hospital Research Institute, Ottawa, Ont. (Moloo); the Department of Prevention and Rehabilitation, University of Ottawa Heart Institute, Ottawa, Ont. (Belanger, Aitken, Mullen); the University of Ottawa Medical School, Ottawa, Ont. (Nadeau); the Ottawa Hospital, Ottawa, Ont. (Foss); and the Department of Anesthesia, University of Ottawa Faculty of Medicine, Ottawa, Ont. (McIsaac).

Competing interests: None declared.

Contributors: J. Sadek, H. Moloo, D. Aitken, L. Williams, I. Raiche, R. Musselman and K.-A. Mullen conceived and designed the study. J. Sadek, H. Moloo, K. Nadeau, K. Foss, L. Williams and K.-A. Mullen acquired the data, which J. Sadek, H. Moloo, P. Belanger, T. Zwiep, D. McIsaac, L. Williams, R. Musselman and K.-A. Mullen analyzed. J. Sadek, H. Moloo, L. Williams and K.-A. Mullen wrote the manuscript. All authors critically revised the manuscript and gave final approval of the article to be published.

Content licence: This is an Open Access article distributed in accordance with the terms of the Creative Commons Attribution (CC BYNC-ND 4.0) licence, which permits use, distribution and reproduction in any medium, provided that the original publication is properly cited, the use is noncommercial (i.e., research or educational use), and no modifications or adaptations are made. See: https://creativecommons. org/licenses/by-nc-nd/4.0/

\section{References}

1. Janz T. Current smoking trends. Ottawa: Statistics Canada; 2012. Available: www150.statcan.gc.ca/n1/pub/82-624-x/2012001/ article/11676-eng.htm (accessed 2020 Jan. 15).

2. Government of Canada. Canadian Tobacco, Alcohol and Drugs Survey (CTADS): summary of results for 2017. Ottawa: Government of Canada; 2017. Available: hwww.canada.ca/en/health-canada/services/ canadian-tobacco-alcohol-drugs-survey/2017-summary.html\#n5 (accessed 2020 Jan. 15).

3. Mullen KA, Manuel DG, Hawken SJ, et al. Effectiveness of a hospital-initiated smoking cessation programme: 2-year health and healthcare outcomes. Tob Control 2017;26:293-9.

4. Turan A, Mascha EJ, Roberman D, et al. Smoking and perioperative outcomes. Anesthesiology 2011;114:837-46.

5. Manuel DG, Perez R, Bennett C, et al. A $\$ 4.9$ billion decrease in health care expenditure: the ten-year impact of improving smoking, alcohol, diet and physical activity in Ontario. Toronto: ICES; 2016.

6. Sørensen LT, Hemmingsen UB, Kirkeby LT, et al. Smoking is a risk factor for incisional hernia. Arch Surg 2005;140:119-23.

7. Andersen T, Christensen FB, Laursen M, et al. Smoking as a predictor of negative outcome in lumbar spinal fusion. Spine (Phila Pa 1976) 2001;26:2623-8.

8. Moreira DM, Aronson WJ, Terris MK, et al. Cigarette smoking is associated with an increased risk of biochemical disease recurrence, metastasis, castration-resistant prostate cancer, and mortality after radical prostatectomy: results from the SEARCH database. Cancer 2014;120:197-204.

9. Bertelsen CA, Andreasen AH, Jørgensen T, et al. Anastomotic leakage after anterior resection for rectal cancer: risk factors. Color Dis 2010;12:37-43.

10. Hatta T, Werthel JD, Wagner ER, et al. Effect of smoking on complications following primary shoulder arthroplasty. 7 Shoulder Elbow Surg 2017;26:1-6.

11. Bullen C. Impact of tobacco smoking and smoking cessation on cardiovascular risk and disease. Expert Rev Cardiovasc Ther 2008; 6:883-95.

12. Godtfredsen NS, Lam TH, Hansel TT, et al. COPD-related morbidity and mortality after smoking cessation: status of the evidence. Eur Respir 7 2008;32:844-53.

13. Saito E, Inoue $M$, Tsugane $S$, et al. Smoking cessation and subsequent risk of cancer: a pooled analysis of eight population-based cohort studies in Japan. Cancer Epidemiol 2017;51:98-108.

14. National Center for Chronic Disease Prevention and Health Promotion (US) Office on Smoking and Health. The health consequences of smoking -50 years of progress: a report of the surgeon general. Atlanta: Centers for Disease Control and Prevention; 2014.

15. Taylor DH, Hasselblad V, Henley SJ, et al. Benefits of smoking cessation for longevity. Am 7 Public Health 2002;92:990-6.

16. Hilleman DE. Intensive smoking cessation intervention reduces mortality in high-risk smokers with cardiovascular disease. Chest 2006;131:446-52.

17. Møller AM, Villebro N, Pedersen T, et al. Effect of preoperative smoking intervention on postoperative complications: a randomised clinical trial. Lancet 2002;359:114-7.

18. Lindström D, Azodi OS, Wladis A, et al. Effects of a perioperative smoking cessation intervention on postoperative complications: a randomized trial. Ann Surg 2008;248:739-45.

19. Nolan MB, Martin DP, Thompson R, et al. Association between smoking status, preoperative exhaled carbon monoxide levels, and postoperative surgical site infection in patients undergoing elective surgery. FAMA Surg 2017;152:476-83.

20. Sørensen LT. Wound healing and infection in surgery: the pathophysiological impact of smoking, smoking cessation, and nicotine replacement therapy: a systematic review. Ann Surg 2012; 255:1069-79.

21. Shannon-Cain J, Webster SF, Cain BS. Prevalence of and reasons for preoperative tobacco use. AANA 7 2002;70:33-40.

22. Glasgow RE, Vogt TM, Boles SM. Evaluating the public health impact of health promotion interventions: the RE-AIM framework. Am 7 Public Health 1999;89:1322-7.

23. Fiore M, Jaen C, Baker T, et al. A clinical practice guideline for treating tobacco use and dependence: 2008. Am 7 Prev Med 2008;35:158-76.

24. Prestwich A, Moore S, Kotze A, et al. How can smoking cessation be induced before surgery? A systematic review and meta-analysis of behavior change techniques and other intervention characteristics. Front Psychol 2017;8:1-14.

25. West R, Hajek P, Stead L, et al. Outcome criteria in smoking cessation trials: proposal for a common standard. Addiction 2005;100:299-303.

26. Sørensen LT, Hemmingsen U, Jørgensen T. Strategies of smoking cessation intervention before hernia surgery - effect on perioperative smoking behavior. Hernia 2007;11:327-33.

27. Lee SM, Landry J, Jones PM, et al. The effectiveness of a perioperative smoking cessation program: a randomized clinical trial. Anesth Analg 2013;117:605-13.

28. Ontario Tobacco Research Unit. Smoke-free Ontario strategy monitoring report. Toronto: Ontario Tobacco Research Unit; 2014.

29. Wu L, Sun S, He Y, et al. Effect of smoking reduction therapy on smoking cessation for smokers without an intention to quit: an updated systematic review and meta-analysis of randomized controlled trials. Int 7 Environ Res Public Health 2015;12:10235-53. 
30. Government of Canada. You can quit smoking. We can help. Ottawa; Government of Canada:2019. Available: https://www.canada.ca/en/ health-canada/services/smoking-tobacco/quit-smoking/tips-help-some one-quit-smoking/you-can-quit-smoking-we-can-help.html (accessed 2020 Jan. 20).

31. Reid RD, Mullen K-A, Slovinec D'Angelo ME, et al. Smoking cessation for hospitalized smokers: an evaluation of the "Ottawa Model". Nicotine Tob Res 2010;12:11-8.

32. Richter KP, Ellerbeck EF. It's time to change the default for tobacco treatment. Addiction 2015;110:381-386.
33. Kuri M, Nakagawa M, Tanaka H, et al. Determination of the duration of preoperative smoking cessation to improve wound healing after head and neck surgery. Anesthesiology 2005;102:892-6.

34. Wong J, Lam DP, Abrishami A, et al. Short-term preoperative smoking cessation and postoperative complications: a systematic review and meta-analysis. Can $\mathcal{F}$ Anaesth 2012;59:268-79.

35. Thomsen T, Villebro N, Møller AM. Interventions for preoperative smoking cessation. Cocbrane Database Syst Rev 2014;2014:CD002294.

36. Herd N, Borland R. The natural history of quitting smoking: findings from the International Tobacco Control (ITC) four country survey. Addiction 2009;104:2075-87. 